\title{
Review of Metamaterials in Microstrip Technology for Filter Applications
}

\author{
Swati Jindal \\ Student, M. Tech. \\ ABES Engineering College, 19th Km stone, $\mathrm{NH}-24$, \\ Vijay Nagar Ghaziabad, 201009 (U.P) India
}

\author{
Jigyasa Sharma \\ Asso. Professor \\ ABES Engineering College, 19th Km stone, $\mathrm{NH}-24$, \\ Vijay Nagar Ghaziabad, 201009 (U.P), India
}

\begin{abstract}
In this work, a review has been presented on filter designs based on microstrip technology with the implementation of metamaterials. It briefly describes the properties of various types of resonators that behave as Left handed materials. Some of the implications of SRRs, CSRRs and other structures in filter design have also been discussed.
\end{abstract}

\section{General Terms}

Metamaterials in microstrip filters.

\section{Keywords}

Metamaterials, microstrip filter, split ring resonator, complementary split ring resonator.

\section{INTRODUCTION}

With fast pace developments and vivid research in the field of metamaterials, they have gained much attention in the field of microwave engineering in recent years. The metamaterials or artificial electromagnetic (EM) materials are those materials that do not have any natural occurrence in nature. They are synthesized artificially through man-made processes. It's not easy to synthesize these materials but they offer such special and distinct qualities that their naturally occurring counterparts are void of. Their structures exhibit strange and uncommon electromagnetic properties. Metamaterial is a material which gains its properties from its structure rather than directly from its composition. These materials are often recorded as left handed materials or double negative materials as they have simultaneous negative permittivity $(\varepsilon<0)$ and permeability $(\mu<0)$. Due to the fact that both $\varepsilon$ and $\mu$ are negative, these materials show negative index of refraction. In 1960's Victor Veselago studied and theoretically suggested these materials [1]. As the name suggests, left-handed materials obey left hand rule which is in complete contrast to the right-hand rule followed for usual materials. The electric field, magnetic field and wave vector of electromagnetic wave propagation in left-handed ( $\mathrm{LH})$ materials are opposite to those in right-handed materials. Their unique properties for instance the reversal of Snell's Law, the Doppler Effect and the Vavilov Cerenkov Effect i.e. radiation produced by a fast moving particle as it travels through a medium have also been anticipated [2]. Since then, these special property materials have contributed extensively in the development of various devices such as antennas, filters, amplifiers and others.

There are a number of resonator types that can be useful in RF and microwave filter applications. The favored resonator types that can be of use are cavity resonators, dielectric resonators, lumped element resonators, transmission line resonators and semi-lumped resonators etc. But in 2005 Chang [3] recommended that the perfect examples of such microwave applications are filters where the transmission line resonators can be effectively used. Metamaterials in filters applications are based on resonant-type metamaterial transmission lines. This helps to obtain a propagating medium with controllable characteristics. Also, the host transmission line combines the sub-wavelength resonators together with elements such as series capacitances or shunt inductances [4]. Few examples of different kinds of sub wavelength resonator used by the resonant type metamaterial transmission lines are: Split-ring resonators (SRRs) as proposed by Pendry et al. in 1999 [5], complementary split-ring resonators (CSRRs) as proposed by F. Falcone et al. in 2004 [6, 7], double slit complementary split ring resonators (DS-CSRRs) and double slit split ring resonators (DS-SRRs) as proposed by R. Marques et al. [8]. A schematic layout of all the four types of resonating metamaterial structures as mentioned above has been depicted in Fig 1 below. In 2006, M. Gil et al. suggested that Characteristic impedance and phase shift are among the chief properties of such transmission lines $[9,10]$. As these resonators are of very small dimensions, they result into compact transmission lines. Once the transmission line has been designed to show certain desired characteristics, its size can be reduced is an important factor suggested by J. Bonache et al. in $2007[11,4]$.

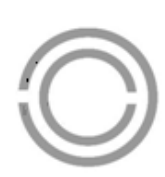

(a)

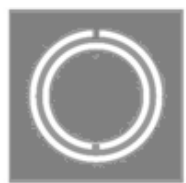

(b)

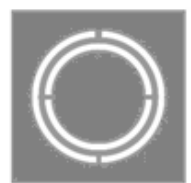

(c)

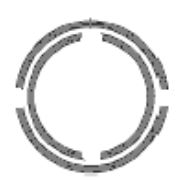

(d)
Fig 1: Schematic layout of (a) SRR, (b) CSRR, and (c) DS-CSRR (metal regions are in dark gray) (d) DS-SRR.

\section{THE SPLIT RING-RESONATORS}

In 2001, Shelby, Smith, and Schultz used arrays of wires (giving rise to negative permittivity) and split-ring resonators (giving rise to negative permeability) at microwave frequencies [12] to experimentally demonstrated first structures used to fabricate the left-handed metamaterials, as shown in Fig 2. Pendry et al. in 1999 [5] proposed Split-ring resonators (SRRs) which is a set of two concentric planar rings with splits in them at opposite ends as depicted in Fig 1(a), printed on a thin dielectric substrate [ $2,5,13]$. The rings are made of nonmagnetic metal like copper and have a small gap between them. The splits in the rings cause the structure to support resonant wavelengths much larger than the diameter of the rings, which cannot occur in closed rings. Thus, SRRs that have a negative index of refraction are 
capable of high-frequency magnetic response [12, 14, 15]. Also, they reveal resonant electric response [15] which is an additional advantage.

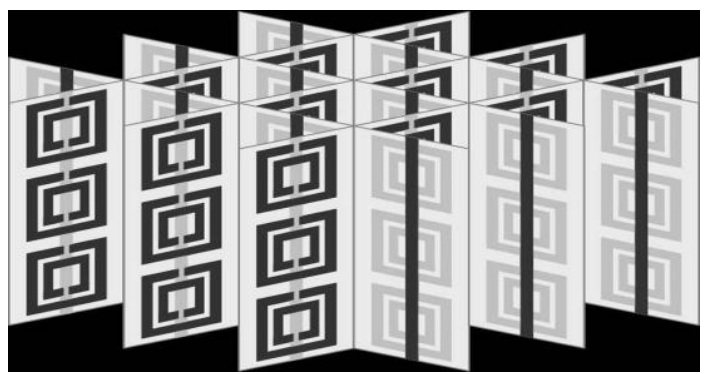

Fig 2: The split-ring resonator and wire medium.

While implementing SRRs using the microstrip technology they are etched above the substrate and close to the conductor strip. Below the resonant frequency the magnetic permeability of a SRR is positive and above resonance frequency it's negative. It is also possible to fabricate a broad-band negative $\mu$, if series gaps are periodically etched in the conductor strip using microstrip technology. SRRs demonstrate a quasi-static resonance by virtue of the distributed capacitance between concentric rings and overall rings inductance. Topology of the SRR and its equivalent-circuit models has been depicted in Fig 3. They act as LC resonators. The same has been proposed in form of the equivalent-circuit model as depicted in Fig 3 [13]. Here in Fig 3, $C_{o}=2 \pi r_{0} C_{p}$, where $C_{o}$ is the total capacitance between the rings and $\mathrm{C}_{\mathrm{p}}$ is the per unit length capacitance between the rings. Also, the resonance frequency is $f_{o}=\left(L_{s} C_{s}\right)^{-1 / 2} / 2 \pi ; C_{s}=C_{o} / 4$ where $C_{s}$ is the series capacitance of the upper and lower halves of the SRR. A SRR is better than the conventional microwave resonator in the sense that it can resonate with a much smaller size, has low radiation losses and very high quality factors.
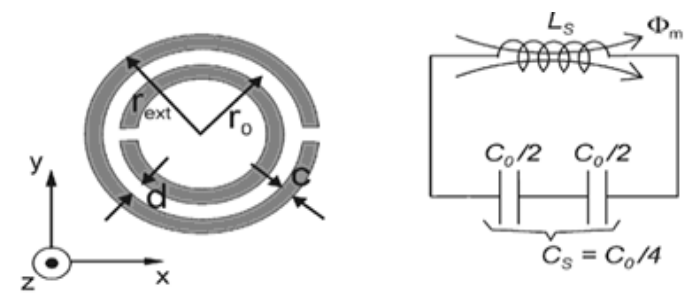

Fig 3: Topology of the SRR and its equivalent-circuit model.

There are various types of split-ring resonators that have been proposed so far, such as rod split rings, nested split rings, single split rings, double split rings, spiral split rings and extended S structures etc. [16]. In the double ring structure, there are different types of split ring resonators while each ring has a gap [17]. A Split Ring Structure can be formed either by using two square rings, one inside the other, or else using concentric circular rings as depicted in Fig 4 and Fig 5 respectively [17]. Another type of such structure is the Symmetrical Ring Structure. Fig 6 shows the unit cell of a symmetrical ring structure. In this, two rectangular shaped rings with gaps had been placed side by side. Both the rings have same size and lie flat such that the gaps face each other [17]. Another example is an Omega Structure having a $\Omega$ shaped ring, as shown in Fig 7 [17]. There are two of these, standing vertical, side by side, instead of lying flat, in the unit cell. Another addition to the group of new metamaterials is a coupled "S" shaped structure $[17,18]$.

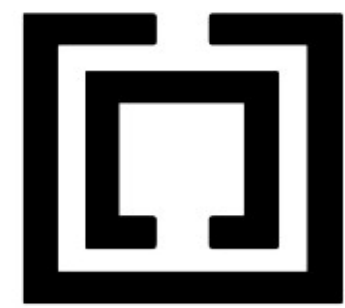

Fig 4: Split Ring structure with square rings.

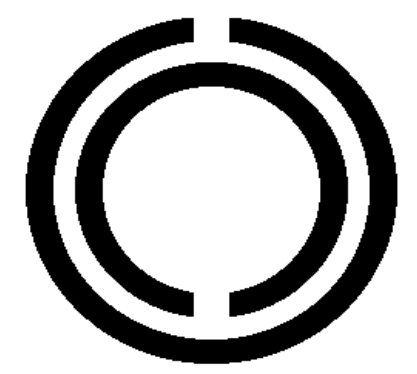

Fig 5: Split Ring structure with concentric circular rings.

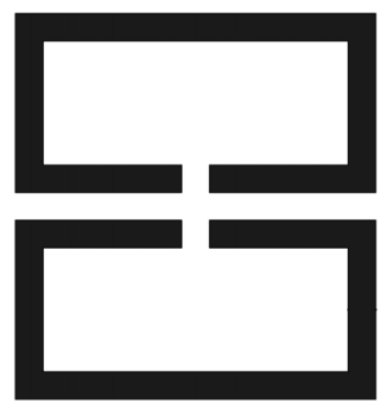

Fig 6: Split Ring structure with Symmetrical Ring structure.

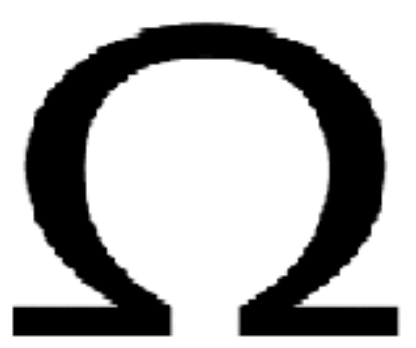

Fig 7: Split Ring structure with Omega structure.

\section{COMPLIMENTRY SPLIT RING RESONATORS}

In 2004, from a duality argument, complementary split ring resonators (CSRRs) were introduced by Falcone et al. as new metamaterial elements that exhibit negative permittivity [6, 7]. A CSRR is the negative image of a SRR etched on the ground plane of substrate, as shown in Fig 1(b), beneath the microstrip. A narrow stop band appeared in the order of the 
resonance frequency of a conventional SRR of identical dimensions etched on the same substrate. Fig 8 depicts the CSRR and its equivalent circuit. On comparing the equivalent circuit of CSRR to that of SRR (Fig 3) it can be well observed that the inductance Ls of the later has been replaced by the capacitance $\mathrm{Cc}$ of a disk of radius $\mathrm{r}_{\mathrm{o}} \mathrm{c} / 2$ surrounded by a ground plane at a distance $\mathrm{c}$ from its edge. Also, the two capacitances $\mathrm{C}_{\mathrm{o}} / 2$, connected in series in the SRR model have now been replaced by two inductances, connected in parallel in CSRR model paving for a connect between inner disk and the ground. Each inductance is given by $\mathrm{L}_{\mathrm{o}} / 2$, where $L_{o}=2 \pi r_{0} L_{p}$ and $L_{p}$ is the per unit length inductance. The first left-handed line based on complementary split-rings resonators was implemented by etching series capacitive gaps in the conductor strip, above the positions occupied by the CSRRs [2]. The series gaps favored the negative effective permeability of the structure. Thus combining these elements (gaps and CSRRs), a narrow band with simultaneously negative permittivity and permeability appeared close to the resonance frequency of the resonators.
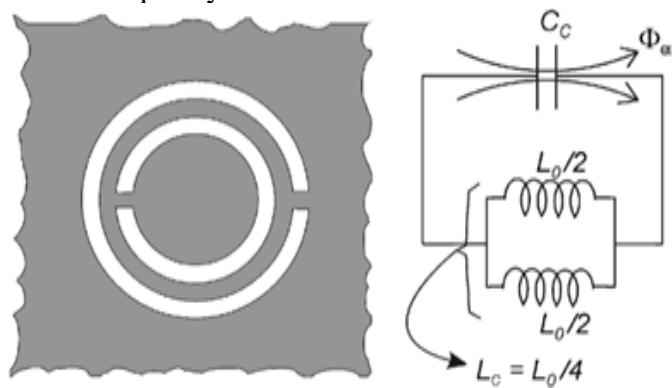

Fig 8: Topology of the CSRR and its equivalent circuit model

A DS-CSRR i.e. Double Slit complementary split-rings resonator as shown in Fig 1(c) can be obtained by introducing two extra slits to a CSRR structure [8]. Also, introducing additional cuts in the edge coupled SRR results in Double Slit split-rings resonator (DS-SRR) [8], as shown in Fig 1(d). The DS-SRR has the same inductance as the SRR but its capacitance is four times smaller as it is the result of the series connected edge capacitances of each SRR quarter. Though this emphasizes the fact that the resonance frequency is twice the resonance frequency of the SRR and as a consequence the electrical size of the particle cannot be made very small. The DS-SRR (or DS-CSRR) has been considered in order to implement moderate or even wideband stop band structures, but these are electrically larger than the SRR (or CSRR) and therefore the accuracy of the circuit model to properly describe lines loaded with such resonators can be argued (19). In 2006, J. Bonache proposed a band pass filter with controllable bandwidth. The band pass filter exploited CSRRs, capacitive gaps, and shorted inductive lines. Also, a band reject filter using CSRR is presented by Falcone in 2003 [8]. Band pass filters integrated with SRRs in coplanar and microstrip technologies have been also successfully deployed $[21,22]$. In microstrip structures, the CSRR has been mainly used for band stop filter design [23]. A varactor loaded band reject tunable filter designed with SRRs is presented by F. Martin et al in 2004 [24].

All the above discussed structures have strengthened that filters constructed from SRRs, CSRRs and DS-CSRRs result in compact sizes.

\section{COMPOSITE RIGHT AND LEFT HANDED TRANSMISSION LINES}

Planar metamaterials can also be implemented by using composite right and left handed transmission lines $(\mathrm{CR} / \mathrm{LH}$ TLs) [25]. CR/LH Transmission Lines have nonlinear phase slopes and the values of CR/LH TL elements can possess two desired impedances at two particular frequencies. These special properties cause a CR/LH TL to be shorter than a conventional right-handed $(\mathrm{RH})$ transmission line. That is why the dimensions of multi-band microwave devices can be reduced using novel transmission lines. CR/LH TLs and SRRs have been used in design of both active and passive microwave devices such as phase shifters, amplifier filters, filters, couplers, and antennas. The CR/LH TL have built in properties of being wideband and low-loss [26] and possibly have wide range of applications. M.Gil et al. in 2007 proposed a wideband band pass filter using CR/LH TLs [27]. In this, complementary split rings resonators (CSRRs) and capacitive gaps edged on the top plane of the substrate are used. In 2004, C. Caloz designed a dual-band filter that is based on CR/LH TLs in which the pass bands of the filter does not necessarily have to be at first and third harmonics [28]. Microstrip filters using SRRs are mainly single-band and also there is scope for research on miniaturization of multi-band filter using $\mathrm{CR} / \mathrm{LH}$ TLs.

Stepped impedance resonator (SIR) is one of the most well known techniques for filter design of planar transmission line resonators used in $\mathrm{RF} /$ microwave applications. That is because SIR is capable of reducing resonator (hence filter) size without degradation of the unloaded-Q and at the same time controls spurious frequencies [29]. In some CR/LH metamaterial filter structures, SIR is used to form a structure which possesses both distinctive characteristics of SIRs and unique properties of CRLH TLs. The composite right and left handed Stepped impedance resonators (CRLH SIRs) are then used to propose novel band pass filters (BPFs) that can either provide enhanced performance or have significantly smaller size while maintaining the integrity of filters' performance. The CRLH SIR with spurious-free stop band response was first proposed by S. Karimian in 2009 [30, 31]. Then, the miniaturized CRLH SIR band pass filter is presented and compared in size and performance to its conventional right handed (RH) counterpart.

\section{METAMATERIAL APPLICATIONS}

\subsection{Band Pass implementations}

In 2005 Gill designed and fabricated a left handed microstrip line with periodic loaded SRRs and metallic vias for the first time [32]. The importance of the structure can be significantly described due to its usage as a narrow band pass filter with fine performance along with circuit size reduction. Gill designed the structure such that, the line is periodically loaded with square shaped SRRs, placed very closely to the conductor strip, and through metallic vias as shown in Fig 9. These metallic via work as if they were a shunt connected inductor. SRRs are modeled by means of a resonant tank with inductance Ls and capacitance Cs, which were coupled to the line through a mutual inductance $\mathrm{M}$. L and $\mathrm{C}$ are the per section inductance and capacitance of the line, respectively, whereas the metallic vias were described by means of shunt connected inductors with inductance $\mathrm{Lp}$. This structure provided the basic platform for filter applications where miniaturization and pass bands are pursued. 
(a)

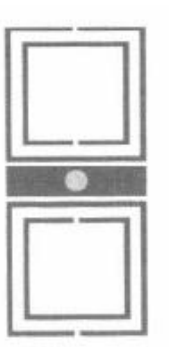

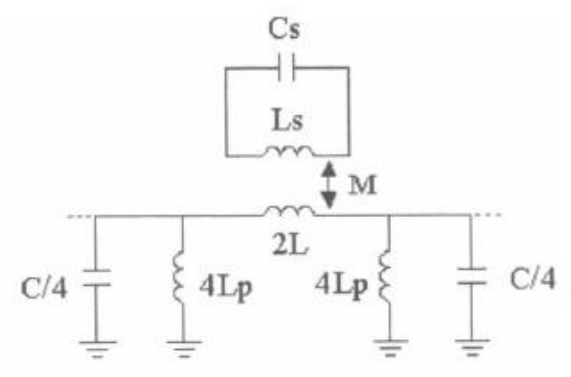

(b)
Fig 9: a) Basic cell of the LHM microstrip line b) equivalent circuit model.

In 2006, a new design approach, based on the use of CSRRs for the synthesis of compact microstrip filters, has been proposed by J. Bonache [33]. This was the first time that planar filters with controllable bandwidth based on CSRRs were achieved. Under this design method, the basic filter cell was designed such as to consist of an arrangement of grounded stubs, CSRRs and series capacitive gaps. The introduction of shunt stubs potentially provided capability to synthesize frequency responses with compact dimensions and controllable BW (bandwidth). An equivalent circuit model for the basic filter cell has shunt stubs and series gaps have been represented by inductors (lumped) and capacitors, respectively and the CSRRs have been modeled by parallel resonant structure. The structure is composed of periodic structure and behaves as a LH-TL with controllable BW.

In $2007 \mathrm{Li}$ Jiusheng proposed, a new kind of a LH metamaterial unit cell based on microstrip technology [34]. The metamaterial unit cell is a combination of a series microstrip gap and a hole for the series capacitor and the shunt inductor, respectively. By using the metamaterial unit cell, a new structure band pass filter is designed and fabricated. Following advantages are then gained from such newly developed band pass filter such as low transmission loss, high return loss, and low fabrication cost.

In 2009, P. K. Singhal has been implemented, a compact microstrip band pass filter using metamaterial resonator [35]. The design is developed with an aim to attain a high level of miniaturization, low cost and ease of fabrication. Narrow bandwidth, low insertion loss and compact dimensions are achieved with the new design. The use of sub-wavelength resonators (in the coupling coefficient method) provides observable reduction in the active area of planar microwave filters. In this type of structures the effective permittivity and effective permeability is negative and fabricated devices are small in dimensions and also show good performance.

\subsection{Ultra Wide Band (UWB) Band Pass implementations}

In 2008 S. Kahng proposed a design for a new UWB band pass filter based upon the microstrip Composite Right and Left Handed Transmission-line (CRLH-TL) [36]. The interdigital coupled lines (Fig 10.)which are much smaller than a quarter wavelength account for the strong capacitive coupling and the grounded stub provide the inductance for the left-handedness. The effective inductance of the interdigital capacitor and the effective capacitance of the short-circuited inductor give rise to the right-handedness characteristics, in order to form an ultra wideband. For improve-ment in size reduction, only 1 unit of the CRLH-TL is taken and implemented with the interdigital line and grounded stubs with their effective parasitics for the UWB. Thus, a realization of component less than a quarter-wavelength is done to result in the achievement of the ultra wide band filtering without the loss of the original advantage of the CRLH-TL.

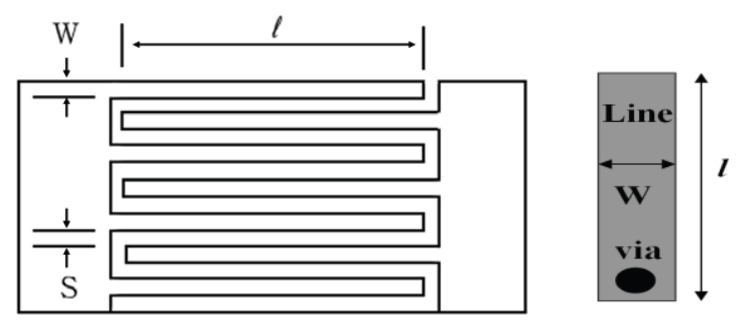

(a)

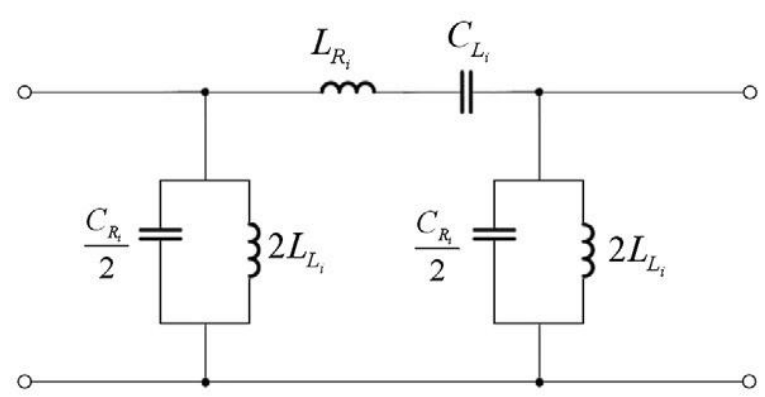

(b)

Fig 10: a) Microstrip inter-digital coupled lines and grounded stub b) Pi-equivalent circuit of the basic unit from the CRLH-TL.

Then in 2009, F. Falcone et al. did an analysis of the frequency response of microstrip lines coupled by CSRRs etched on the ground plane supporting electro-inductive waves (EIWs) [37]. Electro-inductive waves are a powerful medium in designing of ultra wideband filters using metamaterials. By combining SRRs and CSRRs (complementary particle for SRRs) with discontinuous microstrip lines, wideband responses are achievable. The section that provides the wideband coupling is visibly smaller than the coupled-line band pass filters, due to the sub wavelength character of the CSRRs.

\subsection{Band Stop implementations}

In 2004, F.Falcone et al. proposed a structure based on CSRRs that is super-compact [38]. The structure is very compact, exhibits a flat and lossless pass band and produced very high rejection with sharp cutoffs in the forbidden band. A modified version of the CSRR can help in further reduction of the circuit size. This can also be achieved by tailoring the CSRR dimensions. Also, the rejected BW can be increased substantially.

In 2006, Zachary M. Thomas et al. presented a LH metamaterial that consisted of SRRs [39]. The metamaterial consists of the repetition of the symmetric split-ring resonator (SSRR) as shown in Fig 11. The structure is used as a substrate for a microstrip line. In order to realize the metamaterial substrate, the metallic SSRR is printed sixteen times on a dielectric strip. The substrate acts as a natural stop band filter, as is observed through transmission measurements. The region of negative permeability of the substrate corresponds to the stop band in the microstrip. This 
was depicted by the effective parameters obtained for the SRR unit cell. The enhancement of the total bandwidth of a quarter-wave stub stop band filter has resulted by utilizing the natural stop band of the substrate. This design strongly suggested that appropriate designs of microstrip substrate also can be used to improve the existing designs and techniques.

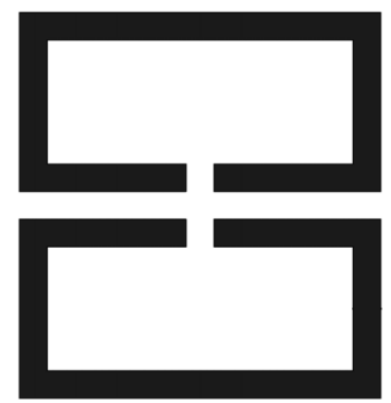

(a)

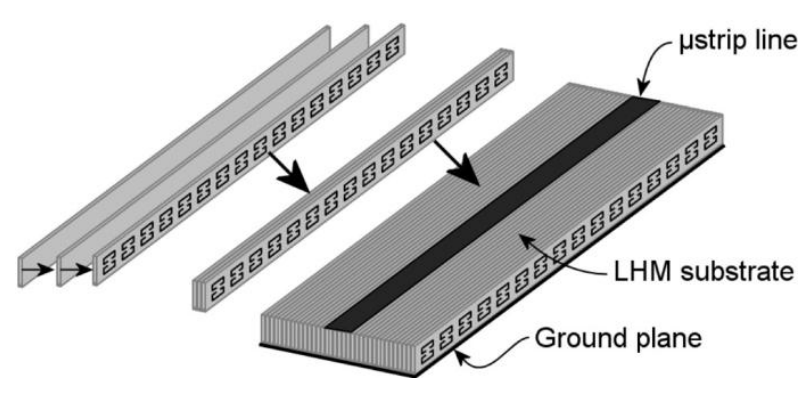

(b)

Fig 11: a) Symmetric split-ring resonator b) metamaterial substrate constructed by combining dielectric strips with SSRRs.

\subsection{Low Pass implementations}

In 2007 Ali Abid designed, fabricated, and characterized a novel maximally flat low-pass filter [40]. The new design structure incorporated CSRRs to yield high filter selectivity and size reduction. An integration of low-pass filter design and the stop-band characteristic of the CSRR is done and implemented. The structure improves the roll-off of a low pass filter, reduces filter size and maintains the pass band performance.

In 2010 Ching-Wen Tang presented a novel microstrip low pass filter with CSRRs [41]. Through proper control of the width of the transmission line and rotating the angle of CSRRs, a wide stop band with sufficient rejection level is achieved. The fabricated filter shows the potential for harmonic suppression with the assistance of CSRRs.

In 2011, Sudhakar Sahu et al. proposed a Compact Metamaterial Microstrip Low-Pass Filter incorporating complimentary hexagonal-omega structures [42]. Such a structure is useful to design compact, low insertion loss (IL), low pass filter with sharp cut-off. The Hex-omega structure is equivalent to an LC resonant tank. The structure improves the roll-off while maintaining the filter pass-band performance. With proper design and loading the hexagonal-omega structure in the ground of the microstrip line reduced the filter size as well along with the improvement in the roll-off of the low pass filter. A notable feature of the design is the ease of fabrication. This ease is due to the facts that, in the design, no via holes or lumped elements are required.

\section{CONCLUSION}

The microstrip filters based on resonant-type left handed and composite right/left-handed metamaterial transmission lines have been revised in this work. Moreover, it has been demonstrated that a metamaterial structure behaves as an effective (continuous) medium with left handed wave propagation in the pass band. Due to the small electrical length of the resonators employed, the presented approaches become very attractive for the design of compact planar microstrip filters using metamaterials. Finally, it is concluded that these microstrip filters using metamaterials are advantageous over conventional microwave filters as they result in improved performance as well as compact sizes.

\section{ACKNOWLEDGMENT}

The authors extend their gratitude towards the entire management and administration of ABES Engineering College for their valuable support. We are also thankful to Mr. Alok Singh, ABES Engineering College for his technical guidance.

\section{REFERENCES}

[1] V.G. Veselago, "The electrodynamics of substances with simultaneously negative values of $\varepsilon$ and $\mu$ ", Sov. Phys. Usp. Vol. 10, pp. 509-514 (1968).

[2] D.R. Smith, W.J. Padilla, D.C. Vier, S.C. Nemat-Nasser, and S. Schultz, "Composite medium with simultaneously negative permeability and permittivity", Phys. Rev. Lett. Vol. 84, 4184 (2000).

[3] Chang, K. Encyclopedia of RF and Microwave Engineering; John Wiley \& Sons, Inc.: Hoboken, NJ, USA, 2005 .

[4] M. Gil, J. Bonache, I. Gil, J. García-García, F. Martín, "Miniaturization of planar microwave circuits by using resonant-type left handed transmission lines",IET Microwave Antennas and Propagation 1 (2007) 73-79.

[5] J.B. Pendry, J. Holden, D.J. Robbins, W.J. Stewart, "Magnetism from conductors and enhanced nonlinear phenomena”, IEEE Transactions on Microwave Theory and Techniques 47 (11) (1999) 2075-2084.

[6] F. Falcone, T. Lopetegi, J. D. Baena, R. Marqus, F. Martn, and M. Sorolla, "Effective negative stop-band microstrip lines based on complementary split ring resonators," IEEE Microwave and Wireless Component Letters, vol. 14 , pp. $280\{282,2004\}$.

[7] F. Falcone, T. Lopetegi, M.A.G. Laso, J.D. Baena, J. Bonache, R. Marqués, F. Martín, M. Sorolla, Babinet principle applied to the design of metasurfaces and metamaterials, Physical Review Letters 93 (2004) 197401-197404.

[8] R. Marques, J. Baena, J. Martel, F. Medina, F. Falcone, M. Sorolla, and F. Martin, "Novel small resonant electromagnetic particles for metamaterial and filter design," in Proceedings of International Conference Electromagnetics in Advanced Applications (ICEAA), Torino, Italy, pp. $439\{443,2003\}$.

[9] M. Gil, J. Bonache, I. Gil, J. García-García, F. Martín, "On the transmission properties of left handed microstrip lines implemented by complementary split rings 
resonators" International Journal of Numerical Modeling: Electronic Networks, Devices and Fields 19 (2006) 87103.

[10] M. Gil, I. Gil, J. Bonache, J. García-García, F. Martín, "Metamaterial transmission lines with extreme impedance values", Microwave and Optical Technology Letters 48 (2006) 2499-2505.

[11] G. Sisó, J. Bonache, M. Gil, J. García-García, F. Martín, Compact rat-race hybrid coupler implemented through artificial left handed and right handed lines, in: Proceedings of the IEEE MTT-S Int'l Microwave Symposium Digest, Honolulu, Hawaii, USA, June 2007, pp. 25-28.

[12] Shelby, RA; Smith, DR; Schultz, S; Smith D.R; Shultz S. , "Experimental Verification of a Negative Index of Refraction", Science 292 (5514)(2001) 77.

[13] R. Marques, F. Mesa, J. Martel, and F. Medina, "Comparative analysis of edge- and broadside-coupled split ring resonators for metamaterial design theory and experiments",IEEE Transactions on Antennas and Propagation, vol. 51, pp. 2572\{2581,2003\}.

[14] Movchan, A. B.; and S. Guenneau, "Split-ring resonators and localized modes", Phys. Rev. B 70(12) (2004) 125116, Retrieved 2009-08-27.

[15] Katsarakis, N.; T. Koschny, M. Kafesaki, E. N. Economou, C. M. Soukoulis, "Electric coupling to the magnetic resonance of split ring resonators", Appl. Phys. Lett. (Crete, Greece and Ames, Iowa, USA: American Institute of Physics) 84 (15) (2004) 2943-2945, Retrieved 2009-09-15.

[16] Moser, H.O. et al; Casse, B.D.F.; Wilhelmi, O.; Saw, B.T. , "Electromagnetic metamaterials over the whole $\mathrm{THz}$ range achievements and perspectives", ELECTROMAGNETIC MATERIALS Proceedings of the Symposium R, ICMAT 2005 (World Scientific Publishing Co.) 18,Retrieved 2009-10-21.

[17] Wu, B.-I.; W. Wang, J. Pacheco, X. Chen, T. Grczegorczyk and J. A. Kong , Gain", Progress in Electromagnetics Research 51(2005) 295-328, Retrieved 2009-09-23.

[18] J. Lezec, Henri; Jennifer A. Dionne, Harry A. Atwater, Frequencies", Science 316 (5823) (2007) 430, Retrieved 2009-10-06.

[19] Miguel Durán-Sindreu, Jordi Naqui, Ferran Paredes, Jordi Bonache and Ferran Martín, "Electrically Small Resonators for Planar Metamaterial, Microwave Circuit and Antenna Design: A Comparative Analysis" Appl. Sci. 2012, 2, 375-395; doi: 10.3390/app2020375.

[20] J. Bonache, I. Gil, J. Garcia-Garcia, and F. Martin, "Novel microstrip band pass filters based on complementary split-ring resonators," IEEE Transactions on Microwave Theory and Techniques, vol. 54, pp. $265\{271,2006$

[21] J. Bonache, F. Marin, F. Falcone, J. Garcia, I. Gil, T. Lopetegi, M.A.G. Laso, R. Marques, F. Medina, and M. Sorolla, "Super compact split ring resonators CPW band pass filters", IEEE MTT-S Int Microwave symp Dig, Fort Worth, TX, June 2004.
[22] I. Gil, J. Bonache, J. Garcia-Garcia, F. Falcone, and F. Martin, "Metamaterials in microstrip technology for filter applications", AP-S Int Symp 1A (2005), 668-671.

[23] J. Garcia-Garcia, F. Martin, F. Falcone, J. Bonache, J.D. Baena, I. Gill, E. Amat, T. Lopetegi, M.A.G. Laso, J.A.M. Iturmendi, M. Sorolla, and R. Marques, "Microwave filters witch improved stop band based on sub-wavelength resonators", Microwave Opt Technology Lett 46 (2005), $283-286$.

[24] I. Gil, J. Garcia-Garcia, J. Bonache, F. Martin, M. Sorolla, and R. Marques, "Varactor loaded split ring resonators for tunable notch filters at microwave frequencies",IEEE Electronic Letters, vol. 40, pp. $1347\{1348,2004$.

[25] J. Garcia-Garcia, J. Bonache, F. Falcone, J.D. Baena, F. Marctın, I. Gil, T. Lopetegi, M.A.G. Laso, A. Marcotegui, R. Marque's, and M. Sorolla, "Steppedimpedance low pass filter with spurious pass band suppression", Electron Lett 40 (2004).

[26] N. Engheta and R. W.Ziolkowski, Metamaterials: Physics and Engineering Explo-rations. Hoboken, NJ: John Wiley\&Sons Inc., 2006.

[27] M. Gil, J. Bonache, J. Garcia-Garcia, J. Martel, and F. Martin, "Composite right/left-handed metamaterial transmission lines based on complementary split-ring resonators and their applications to very wideband and compact filter design", IEEE Transactions on Microwave Theory and Techniques, vol. 55, pp. 1296\{1304, 2007.

[28] I. Lin, M. DeVincentis, C. Caloz, and T. Itoh, "Arbitrary dual-band components using composite right/left-handed transmission lines", IEEE Transactions on Microwave Theory and Techniques, vol. 52, pp. 1142\{1149, 2004.

[29] E. G. Cristal and S. Frankel, "Hairpin-line and hybrid hairpin line/ half-wave parallel-coupled-line filters," IEEE Trans. Microwave Theory Tech., vol. MTT-20, Nov. 1972, pp. $719-728$

[30] S. Karimian and Z. Hu, "Miniaturised left-handed stepped impedance resonator for WLAN applications," 3rd IEEE International Symposium on Microwave, Antenna, Propagation (MAP) and EMC Technologies for Wireless Communications, 27- 29 October 2009, pp. 111-113.

[31] S. Karimian, Z. Hu, "Left-handed tri-section stepped impedance resonator," IEEE International Symposium on Radio-Frequency Integration Technology, 2009, pp. 311-314.

[32] Gil, I., J. Bonache, J. Garcia-Garcia, F. Falcone, and F. Martin, "Metamaterials in microstrip technology for filter applications", 2005 IEEE Antennas and Propagation Society International Symposium, Vol. 1A, 668\{671, $3\{8$ July, 2005.

[33] J. Bonache, I. Gil, J. García-García, and F. Martín, "Novel microstrip bandpass filters based on complementary split rings resonators", IEEE Trans. Microw. Theory Tech., vol. 54, no. 1, pp. 265-271, Jan. 2006.

[34] Li Jiusheng and Du Tieying, "Application of metamaterial unit cell in bandpass filter", Microwave and Optical Technology Letters, Vol. 49, No. 9, 2007, pp. 2109 - 2111. doi: 10.1002/mop.22659. 
[35] R. N. Baral and P. K. Singhal, "Miniaturized Microstrip Band pass Filter Using Coupled Metamaterial Resonators," International Journal Of Microwave and Optical Technology, Vol. 4, No. 2, 2009.

[36] S. Kahng and J. Ju, " Design of the UWB bandpass filter based on the 1 cell of microstrip CRLH-TL", Proc. International Conference on Microwave and Millimeter Wave Technology (IEEE-ICMMT 2008), Vol. 1, pp.6972,2008

[37] M. Navarro-C'Ia, J. M. Carrasco, M. Beruete, And F. Falcone, "Ultra-Wideband Metamaterial Filter Based On Electroinductive-Wave Coupling Between Microstrips", Progress In Electromagnetics Research Letters, Vol. 12, Pp. 141-150, And 2009

[38] F.Falcone, T.Lopetegi, J.D Baena, R. Marques, F. Martin and M. Sorolla,"Effective Negative Stopband Microstrip Lines Based on Complementary Split Ring Resonators", IEEE Microwave And Wireless Components Letters, Vol. 14, No. 6, pp.1522-1525, June 2004 .May 2006.
[39] Zachary M. Thomas, Tomasz M. Grzegorczyk, Bae-Ian $\mathrm{Wu}$, and Jin Au Kong, "Enhanced Microstrip Stopband Filter Using A Metamaterial Substrate", Microwave and Optical Technology Letters, vol. 48,no.8 pp. 141-150, 2009.

[40] Ali Abid and Hu Zhirun, "Sharp Cut-Off, Miniaturized Metamaterial Binomial Microstrip Low-Pass Filter",Microwave and Optical Technology Letters, Vol. 49, No. 10, Oct 2007, pp. 2406-2409, DOI: $10.1002 / \mathrm{mop} .22799$.

[41] C.W.Tang and S.-C.Yang, "Employing Complementary Split-Ring Resonators for the Wide Stopband Microstrip Lowpass Filter Design", Microwave and Optical Technology Letters, Vol. 52, No. 11, 2010, pp. 25922594. doi:10.1002/mop.25541.

[42] Sudhakar Sahu, Rabindra Kishore Mishra, Dipak Ranjan Poddar, " Compact Metamaterial Microstrip Low-Pass Filter", Journal of Electromagnetic Analysis and Applications, 2011, 3, 399-405, doi:10.4236/jemaa.310063. 